\title{
«Competencia asistida» por el lugar y la consolidación de la segregación residencial de gran escala: Lima, 1993-2007
}

\author{
OMAR PEREYRA* \\ Pontificia Universidad Católica del Perú \\ pereyra.o@pucp.pe
}

https://doi.org/10.18800/rcpg.201801.002

\section{RESUMEN}

En este artículo se explora la relación entre incentivos detrás de las decisiones respecto al lugar de residencia y los patrones de segregación que estos generan. Comparando datos de los censos de 1993 y de 2007, encontramos dos grandes modelos de producción de la ciudad. Uno de "competencia segmentada", correspondiente al periodo de crecimiento por migraciones sobre la base de una clara diferenciación entre un mercado formal e informal de tierras. Otro de "competencia asistida", correspondiente al periodo posterior a las reformas estructurales neoliberales en donde las preferencias residenciales individuales se complementan con iniciativas inmobiliarias público-privadas dirigidas a los sectores medios. Consecuencia de este nuevo modelo es que la segregación de gran escala se consolida en la ciudad y se convierte en una de sus características principales. Finalmente, encontramos que, si bien la microsegregación sería una dinámica presente en Lima, esta sería un fenómeno menor.

Palabras clave: urbanización, segregación residencial, Lima.

+ Recibido el 06 de febrero de 2018; aceptado el 22 de mayo de 2019.

* Doctor en Sociología por Brown University. Profesor asociado del Departamento de Ciencias Sociales de la Pontificia Universidad Católica del Perú. 


\section{«Assisted competition» for place and the consolidation of great-scale segregation:} Lima, 1993-2007

\section{Abstract}

I explore the relationship between incentives behind residential choices and the segregation patterns that they generate. By comparing census data from 1993 and 2007, I find two main models of production of the city. One of "segmented competition" which corresponds to the period of growth by migration with a clear differentiation between the formal and informal land markets. Another of "assisted competition" which corresponds to the period after neoliberal structural reforms, where individual residential preferences are fostered by private-public housing initiatives clearly targeted to the middle sectors. A consequence of this new model is that great scale segregation consolidates in the city and becomes one of its main characteristics. Finally, I find that, despite the fact that micro-segregation is found in Lima, it is a marginal phenomenon.

Keywords: urbanization, residential segregation, Lima. 


\section{INTRODUCCIÓN}

Desde hace ya algunas décadas, la segregación residencial ha empezado a ser un tema de interés entre los investigadores de las ciudades latinoamericanas (Telles, 1992; Sabatini, 2003; Roberts y Wilson, 2009; Sabatini, Wormald y Rasse, 2013; Marques, 2016). El fenómeno es relevante pues se sabe que la segregación residencial puede contribuir a la reducción de oportunidades y a la pobreza. En el Perú, y en Lima en particular, la segregación residencial ha empezado a ser medida para entender su magnitud y para describir sus cambios en el tiempo (Pereyra, 2006; Peters y Skop, 2007; Fernández de Córdova, 2012; Fernández de Córdova, Fernández Maldonado y Del Pozo, 2016; Herrera, Benavides, Etesse y Letoutier , 2016). También se ha empezado a explorar su efecto en la calidad educativa (Peters y Hall, 2004), en la violencia familiar (Benavides, León, Paredes y La Riva, 2015) y en el logro ocupacional (Benavides et al., 2019). Este artículo se enfoca en la forma cómo se produce la segregación residencial. $\mathrm{O}$ de forma más específica, se pregunta por la forma en que las dinámicas urbanas moldean o generan nuevos incentivos respecto a las decisiones residenciales, las cuales a su vez refuerzan o cambian los patrones de segregación existentes. Al enfocarnos en la dinámica de los distintos actores o grupos (y no solo en los pobres o en los llamados sectores minoritarios), nos alejamos del tema clásico de la relación entre la concentración de la pobreza y la reproducción de la pobreza.

Para este artículo se usa a Lima como caso de estudio. El presentar un caso nos permite indagar con mayor detalle cómo grandes procesos comunes a las ciudades latinoamericanas toman una forma particular en una metrópoli. Lima no es ni un caso representativo ni uno atípico. Sin embargo, la fuerza con que experimenta algunos procesos contemporáneos comunes a la región —como el ser una de las economías de mayor crecimiento, el tener un crecimiento significativo de las clases medias, el proceso de formalización del mercado de vivienda y el nuevo impulso inmobiliario-, la hacen un caso atractivo y pertinente para explorar la interconexión entre estos procesos y sus consecuencias.

Sugerimos los términos "competencia segmentada» y "competencia asistida» para describir los modelos de toma de decisión residencial en el periodo de urbanización por migración y en el periodo neoliberal, respectivamente. "Competencia segmentada» se refiere a un modelo en el que existen dos modos principales de tomar decisiones respecto al lugar de residencia en un contexto de mercado dual (formal e informal), el cual aparece como el gran mecanismo diferenciador respecto a la ubicación a la que uno puede aspirar en la metrópolis, 
separando progresivamente a los más ricos de los más pobres. «Competencia asistida» se refiere a un modelo que se superpone al anterior, pero en el cual una coalición pública-privada favorece el acceso a vivienda para un grupo específico (los sectores medios) y fomenta la ubicación de este grupo en un espacio particular de la ciudad, contribuyendo con ello a la intensificación del modelo de segregación residencial de gran escala.

\section{REVISIÓN DE LA LITERATURA}

Se entiende por segregación residencial al fenómeno por el cual personas que pertenecen a grupos determinados se separan geográficamente de otros grupos en una ciudad. Este fenómeno ha llamado la atención de investigadores en distintas ciudades pues se ha encontrado que el mismo contribuye, a través de un conjunto de dinámicas o mecanismos específicos, a reducir o aumentar las oportunidades de vida de las personas o grupos, y en particular la reproducción de la pobreza. A diferencia de las explicaciones que privilegian las características de las personas o grupos (su capital humano, nivel educativo, recursos, etcétera) o de explicaciones más estructurales que privilegian dinámicas macro (como la estructura de clases, la cultura o los arreglos institucionales), el estudio de la segregación residencial se encuentra en un nivel meso, específicamente en los efectos del contexto espacial (vecindarios) sobre las personas.

La agenda principal de los estudios sobre segregación residencial se ha centrado en delinear mecanismos específicos por los cuales las características de los vecindarios afectan las oportunidades de vida de las personas. Literatura sobre la "geografía de oportunidades» (Galster y Killen, 1995), «efecto concentración» (Wilson, 1987), «estigma del lugar» (Wilson 1997; Tilly, Moss, Kirschenman y Kennelly, 2001) y «efectos-barrio» (Sampson, Morenoff y Ganon-Rowley, 2002) son relevantes al respecto. Sin embargo, es poco lo que se ha discutido respecto a la producción de la segregación residencial. Existen posiciones que se enfocan en las preferencias individuales, las cuales, de forma agregada, generan el fenómeno mayor de la segregación (Schelling, 1971). Cuentan con mayor aceptación las posiciones de tipo más estructural, sea por la dinámica propia del mercado capitalista (Castells, 1974; Harvey, 1978), las que ponen el énfasis en la desindustrialización y los cambios en el Estado de bienestar (Wilson, 1987; Wacquant, 2008), o las que ponen el énfasis en la consistencia del racismo en los distintos actores del mercado de vivienda y en las políticas de los gobiernos de las ciudades (Massey y Denton, 1993; Charles, 2006). En el caso de América Latina la literatura resalta el rol de la lógica del mercado capitalista (Abramo, 2012), así como una conexión 
entre reformas estructurales y una intensificación de la segregación residencial y de sus efectos (Katzman, 2001; Roberts y Wilson, 2009). No se cuenta, sin embargo, con estudios sobre la toma de decisiones individuales, ni (con información de primera mano) sobre el papel de los actores inmobiliarios o del Gobierno al respecto (pero ver Benzús, 2018). Por otro lado, se empieza también a señalar como una tendencia importante la aparición de un nuevo modelo de segregación residencial, la microsegregación, encarnada en fenómenos como la aparición de condominios cerrados de elite y la gentrificación. Ambos fenómenos, de acuerdo a la literatura existente, generarían una menor distancia espacial entre grupos, pero no necesariamente disminuirían la distancia social entre los mismos, ni facilitarían su interacción y menos aún su integración (Caldeira, 2000; Janoschka, 2002; Thiebert y Osorio, 2014; pero ver Cáceres y Sabatini, 2004).

Respecto a esta discusión, tema relevante es la particularidad de las ciudades latinoamericanas respecto a la estructura dual (formal e informal) en la base de su urbanización. Siguiendo a Abramo (2012, pp. 36-37), dos grandes lógicas son comunes en la producción de las ciudades. 1) Una «formal», que tiene dos variantes: la del mercado, que se basa en las transacciones monetarias y que toma forma en el contrato de compra-venta; y la del Estado, que se basa en la membresía en una comunidad o ciudadanía y que toma la forma de vivienda social o de concesión de terrenos. 2) Una «informal», que ocurre al margen del mercado y del Estado, y que se ampara en una «lógica de la necesidad» que lleva a la toma de tierras en sus distintas formas (barriadas, invasiones, favelas, poblaciones, entre otras).

La vía informal (invasión seguida de autoconstrucción) ha sido una lógica relevante en la producción de las ciudades latinoamericanas. En Lima, Calderón estima que para 1998 el 56\% del suelo urbanizado tiene un origen informal o ilegal (2005, p. 39). Sin embargo, en la medida en que la ubicación de algunos de estos asentamientos se hace deseable, estos adquieren un precio y se insertan en el mercado (formal o informal). Del mismo modo, han existido políticas de formalización que han contribuido a que algunas de estas áreas se inserten al mercado inmobiliario. En este sentido, si bien la producción informal es parte de la estructura sobre la que se levanta la urbanización de Lima - y esta sigue desarrollándose en zonas nuevas de invasión o en un nuevo mercado de alquiler (informal) en zonas informales y formales-, esta cede terreno ante el proceso de formalización de estos espacios y la nueva producción formal del espacio (horizontal y más recientemente vertical).

En este artículo se propone un marco para entender el fenómeno de la producción de la segregación, el cual, desde dinámicas macro y con el empuje de actores 
institucionales, incentiva determinadas decisiones y acciones individuales o familiares respecto al lugar de residencia. Estas decisiones, de forma agregada, generan patrones mayores de segregación residencial. Se puede hablar entonces de la producción de una "estratificación de lugares» por los cuales los actores compiten para mantener o mejorar sus oportunidades de vida (Logan y Molotch, 1987).

Parte importante de nuestro trabajo es medir la segregación residencial en dos momentos (1993 y 2007) para ver el desarrollo de este fenómeno durante quince años de reformas estructurales. Discutiremos, a partir de estos resultados, la relevancia de la tesis de la microsegregación para Lima. Consideramos, además, que las diferencias en los índices de segregación residencial no pueden ser interpretadas de forma descontextualizada. Lima de 1993 es radicalmente distinta a Lima de 2007. Han cambiado el mercado de vivienda, su gobierno y la forma de tomar decisiones de parte de los actores. Por ello, nos interesa en particular el asociar dos momentos en la segregación residencial con dos formas de tomar decisiones en los mercados de vivienda.

\section{MÉTodos}

En este artículo se procede de la siguiente forma: 1) Se busca reconstruir las motivaciones o incentivos respecto a las decisiones residenciales a partir de los principales procesos urbanos existentes para las etapas de la urbanización por migración (1940-1990) y para la ciudad neoliberal (1992 a la actualidad). Ello se hace sobre la base de la revisión de la literatura sobre el caso de Lima. 2) Se describen los modelos de segregación residencial producida para ambas etapas. Para ello, se usa información a nivel distrital de los censos de 1993 y 2007. Los datos del censo de 1993 nos dan una buena imagen de la ciudad de Lima al inicio de estas reformas (iniciadas en 1992). Los datos del censo de 2007 nos dan una buena imagen del camino que toma la producción de la ciudad luego de quince ańos de reformas estructurales. Lamentablemente, los datos del censo de 2017 aún no se encuentran disponibles a nivel de análisis y para las variables que permitirían la comparación en el tiempo.

En el análisis se divide a la población de Lima en tres grupos por «nivel socioeconómico» (NSE). Dichos grupos están definidos sobre la base del estimado de ingresos que calculó el Instituto Nacional de Estadística e Informática (INEI) para los censos de 1993 y 2007. Estos datos vienen de dos fuentes: 1) la base de datos a nivel manzana para el censo de $1993^{1}$ y 2) la información

1 La información correspondiente al censo 1993 proviene de la Base de Datos del Censo Nacional de Población y Vivienda 1993. 
publicada en los planos distritales a nivel socioeconómico del INEI para el censo 2007 (INEI, 2009)2. Los rangos de ingresos fueron calculados por el INEI sobre la base de un conjunto de variables sobre calidad de la vivienda, artefactos poseídos por el hogar, nivel educativo de los miembros del hogar y dependencia económica ${ }^{3}$. Si bien el INEI presenta originalmente cinco NSE, para facilitar la presentación de los datos en este artículo, dichos NSE se reagruparon en tres categorías: bajo, medio y alto.

Se usan indicadores globales estándar para medir la segregación residencial, como el de disimilaridad $(D)$, aislamiento $\left(P^{*}\right)$ y aglomeración ( $I$ de Moran). Cada indicador mide distintas características de la segregación. El índice de disimilaridad $(D)$ mide, en un rango de 0 a 1 , el porcentaje de población que debería reubicarse o moverse de su lugar de residencia para alcanzar una distribución poblacional no-segregada. Un valor de 0 indica que la población de un grupo se encuentra ubicada de una forma balanceada en el espacio de tal forma que el fenómeno de la segregación residencial no existe. Un valor de 1 indica que toda la población de un grupo debiera ser reubicada en el espacio para diluir la segregación. El índice de aislamiento $\left(P^{*}\right)$ mide, en un rango de 0 a 1 , el grado en que la población de un grupo está rodeada (residencialmente) por miembros de su mismo grupo. Un valor de 0 indica que los miembros de un grupo se encuentran completamente rodeados de miembros de otros grupos, mientras que un valor de 1 indica que los miembros de un grupo se encuentran complemente rodeados de miembros de otros grupos. El índice $I$ de Morán, en cambio, va de -1 a 1 , en donde un valor de -1 indica que no existen aglomeraciones de población de un tipo en el espacio, mientras que un valor de 1 indica que toda la población de un grupo se encuentra completamente concentrada en un área de la ciudad.

Los indicadores de segregación muestran dos problemas principales. El primero es que se basan en el lugar de residencia, dejando de lado o no pudiendo medir el contacto entre grupos en otros espacios distintos a la residencia o cuando se desplazan; por ejemplo: en el trabajo, en los centros educativos, en el tiempo de ocio o en los espacios mismos de la movilidad. A pesar de que es de suponer que el contacto entre grupos existe en estos espacios, la literatura

\footnotetext{
2 Si bien la información presentada por el INEI 2009 (censo 2007) se encuentra presentada en mapas a nivel manzana y por distritos, esta información no está disponible al público en la forma de una base de datos. Sin embargo, la publicación del INEI sí presenta el dato absoluto de hogares por NSE para cada distrito.

3 Para mayor detalle sobre el cálculo de estos rangos de ingresos, ver la sección metodológica de INEI 2009.
} 
sobre segregación residencial, sobre efectos barrio y sobre geografía de oportunidades convergen en indicar que el lugar de residencia (sobre todo si este es en un lugar segregado) es un factor central en la disminución o aumento de las oportunidades de vida.

El segundo problema, más específico para nuestro caso, es el tamaño de las unidades con las que se trabaja para el cálculo de estos índices. Este es un asunto considerable para nuestro trabajo en dos aspectos: 1) algunos distritos son bastante más grandes que otros, generando problemas de comparación entre estos; 2) siendo el distrito la unidad de análisis, se tiene que asumir que en su interior los miembros de distintos grupos se encuentran distribuidos de forma equilibrada (no segregada) en el espacio. Por ello, la información a nivel distrital es gruesa para detectar la presencia y ubicación de formas finas de segregación residencial ${ }^{4}$.

Sin embargo, la información a nivel distrital es la única información disponible de modo libre hasta el momento. Al nivel de análisis al que trabajamos, solo podemos indicar si la segregación residencial (sobre la base de datos distritales) ha aumentado o disminuido para la ciudad; pero no podemos decir cuáles son las áreas segregadas de la ciudad. Aun así, es posible inferir que de acuerdo al cambio de composición de población de los distritos en el periodo 1993-2007, estos se tornan más homogéneos, más heterogéneos o si se mantienen relativamente iguales. Pero no podemos decir mucho respecto a si los grupos que se encuentran en un distrito estarían espacialmente dispersos o aglomerados en algunas áreas del mismo. Es decir, no podemos decir si la segregación ha aumentado o disminuido al interior de estos distritos. Aunque sí podemos evaluar su nivel de impacto de la segregación de pequeña escala en la ciudad: en términos generales, si la microsegregación (condominios cerrados o gentrificación) fuera un proceso mayor, los distritos más pobres de Lima o del centro histórico de la ciudad en 1993 pasarían a ser más heterogéneos en 2007. Del mismo modo, valores altos en nuestros índices indicarían altos niveles de homogeneidad social; es decir, una mayor presencia de la segregación de gran escala. Mientras que la disminución en sus valores sería indicador de mayor heterogeneidad, es decir de una mayor presencia de microsegregación o de la disolución de la segregación.

\footnotetext{
4 Compárese, por ejemplo, con los trabajos de Fernández de Córdova et al. (2016) y de Herrera et al. (2016), en los cuales se mide la segregación residencial en Lima de 1993 a 2007 con información a nivel zona y a nivel manzana, respectivamente. Sin embargo, el énfasis de estos trabajos se encuentra en la medición y descripción de la segregación residencial.
} 


\section{Dinámica de LA CIUdAd EN El PERIOdo de LAS gRANDES MigRaciones}

$\mathrm{Al}$ igual que otras ciudades latinoamericanas, Lima empezó a crecer de forma acelerada hacia la década de 1940 debido a la migración del campo a la ciudad. Dicha migración fue incentivada tanto por la transición demográfica que afectó al campo expulsando población, como por la industrialización y el aumento de servicios en la ciudad que atrajeron población (Roberts, 1978; Gilbert, 1998).

Volviendo a Lima, la estructura centralista de la ciudad jugó un rol central respecto a las decisiones residenciales. Inicialmente, buena parte de los empleos y servicios distintos se concentraban en el centro de la ciudad. Sin embargo, surgieron problemas asociados a ello: la densificación del centro generó un conjunto de externalidades negativas, como la tugurización y deterioro de viviendas, la criminalidad, la contaminación, el ruido, entre otros. La forma de responder a estas externalidades fue diferenciada para los grupos sociales. Lima, al igual que otras ciudades latinoamericanas, desarrolló una estructura dual (Calderón, 2005): un mercado formal de vivienda (para los trabajadores formales, las clases medias y altas) que se extendió desde el centro histórico hacia una dirección, formando lo que Sabatini (2003) llama un «cono de alta renta» (en el caso de Lima, hacia el sur-este del centro); y un mercado informal de vivienda (para las olas sucesivas de migrantes) hacia las otras direcciones de la ciudad (en el caso de Lima, los llamados «conos»).

Debe resaltarse que, en esta estructura dual, las motivaciones y costos que guiaron a las personas en sus decisiones residenciales eran distintos. El principal mecanismo diferenciador para entrar en una de estas dos grandes áreas de la ciudad era el contar con los recursos disponibles para participar en uno $\mathrm{u}$ otro lado del mercado de vivienda. A partir de esta posición, las familias desarrollaron estrategias residenciales diferenciadas. Proponemos usar el término "competencia segmentada» para describir el modelo de toma de decisiones residenciales para esta etapa. Usamos el término «competencia» (y no «conflicto») pues se trata más bien de decisiones individuales o familiares por maximizar sus oportunidades de acuerdo a sus recursos disponibles. Usamos el adjetivo «segmentada» pues se trata de una competencia que se enmarca en una estructura social mayor que asigna recursos diferenciados y, a partir de la posesión de los mismos, invita a los individuos o familias a ubicarse en zonas distintas de la ciudad: en la zona de crecimiento formal o en la zona de crecimiento informal5.

\footnotetext{
5 La idea del mercado dual o segmentado de vivienda es, claro está, una simplificación: existen invasiones, tugurios y zonas rurales en la zona de expansión del mercado formal (zona sur-este), del mismo modo que existen en la zona de expansión periférica desarrollos urbanos que no son informales, como
} 
En adelante, las zonas de la ciudad (y las familias a su interior) formarán contextos distintos y trayectorias desiguales.

Aquellos que contaban con los recursos para participar en el mercado formal de vivienda podían elegir, de acuerdo a sus recursos disponibles, viviendas y lugares de distintos precios. El tamaño de la vivienda, su ubicación, el estatus del lugar y la distancia al centro de la ciudad eran factores importantes a la hora de evaluar las opciones. La disponibilidad de transporte público o privado, en buena cuenta, permitía vivir en lugares cada vez más alejados y exclusivos, pero ello significaba también un costo económico a evaluar. De este modo, en este espacio de la ciudad, a medida que el centro y sus áreas contiguas fueron tugurizándose, los sectores más ricos fueron alejándose paulatinamente buscando mejores lugares para vivir, pero en parte también buscando una mayor distancia de otros grupos considerados como indeseables (Parker 1995). En su camino fueron seguidos por los sectores medios y luego por los sectores populares. De este modo, esta área de la ciudad se desarrolló siguiendo una lógica similar a la dinámica de «expansión»e «invasión-sucesión» sugerida por la antigua Escuela de Chicago.

Por otro lado, para los que no podían participar en el mercado formal de vivienda, la opción era ingresar a la otra área de la ciudad (los Conos Norte, Este y Sur). El principal mecanismo no fue la compra de vivienda, sino la invasión de terrenos y la autoconstrucción (Driant, 1991; Riofrío, 1991; Calderón, 2005). En este caso, los criterios principales para la elección de vivienda eran no solo la distancia al centro (algo deseable por la proximidad a los centros de empleo), sino también el momento de llegada a la ciudad. De este modo, las olas más antiguas de migrantes se ubicaron en las zonas más cercanas al centro de la ciudad; mientras que las nuevas olas de migrantes, en zonas cada vez más distantes. Es decir, mientras todos buscaban vivir lo más cerca posible al centro, el margen de elección estuvo limitado por la presencia de grupos que tomaron posesión previa de estos lugares.

Con el tiempo, se fueron asentando dinámicas de diferenciación al interior de estas zonas (Pereyra, 2006; Fernández de Córdova, 2012). Por un lado, en la zona formal (la zona sur-este) la dinámica de «invasión-sucesión» generó que esta tuviera niveles considerables de heterogeneidad social; aunque de todos modos podía notarse una tendencia clara en la cual una mayor distancia del

zonas de mercado formal en estas áreas (urbanizaciones y otras), además de zonas cedidas por el Estado y asentamientos humanos planificados. Sin embargo, a pesar de ser una simplificación, señala una tendencia principal dentro del crecimiento de la ciudad. 
centro de la ciudad estaba asociada a mayor presencia de población de estratos medios y altos; y la mayor cercanía al centro, con la presencia de algunos bolsones de pobreza. Por otro lado, en los Conos la situación era inversa: zonas más cercanas al centro estaban relacionadas con olas más antiguas de migración $y$, en consecuencia, se podía encontrar en ellos mayores niveles de consolidación urbana (incluyendo la formalización de varios de estos barrios) y menor pobreza.

Por el contrario, las nuevas olas de migración (la población más pobre) fueron asentándose en zonas cada vez más distantes de la ciudad. Dos elementos complejizan un poco más esta dinámica. Por un lado, con el tiempo, las invasiones antiguas consiguieron la formalidad y un mejor acceso a servicios básicos. Ello jugó a favor de que estas zonas empiecen a tener un valor en el mercado formal de vivienda, pero además que empiecen a ser deseables comercialmente (Driant, 1991). Se une a ello un proceso de movilidad social ascendente de varias de estas familias, el cual hizo que varias de estas zonas pasen de ser pobres a ser más heterogéneas. Por otro lado, los jóvenes nacidos en las zonas de invasión más antiguas (las más cercanas al centro), empezaron a buscar lugares de vivienda para sus nuevas familias. En algunos casos, optaron por las ventajas que ofrecían sus barrios de origen, como la cercanía al centro y el mantenerse cerca a sus padres. Optaron así por construir segundos y terceros pisos en las viviendas originales de sus padres para vivir con sus nuevas familias (Riofrío, 1991). En otros casos, prefiriendo el bajo costo de conseguir una vivienda nueva por invasión, se unieron a los nuevos migrantes más pobres y optaron por invadir las nuevas zonas más lejanas o las zonas no invadidas anteriormente (las zonas más empinadas de los cerros) dada la dificultad del terreno (Barreda y Ramírez, 2004).

Así se creó en Lima, al igual que en otras ciudades de América Latina, un modelo que Sabatini (2003) llama «segregación a gran escala». Son características saltantes de este modelo un continuo crecimiento horizontal de la ciudad, la gran distancia espacial entre sectores alto y bajo, y niveles considerables de heterogeneidad en la zona central de la ciudad. En el caso de Lima, las áreas más lejanas de los Conos alojaban grandes concentraciones de población de NSE bajo (Mapa 1A); la zona central de la ciudad (tanto la formal como la informal) exhibía una relativa concentración de población de NSE medio (Mapa 1B); y ningún distrito que concentre a la población de NSE alto (Mapa 1C) ${ }^{6}$.

6 Aunque a nivel de zonas censales sí se logran ubicar algunos bolsones de NSE alto hacia el sur-este. Ver Pereyra, 2006. 
Mapa 1. Población por nivel socioeconómico, Lima 1993

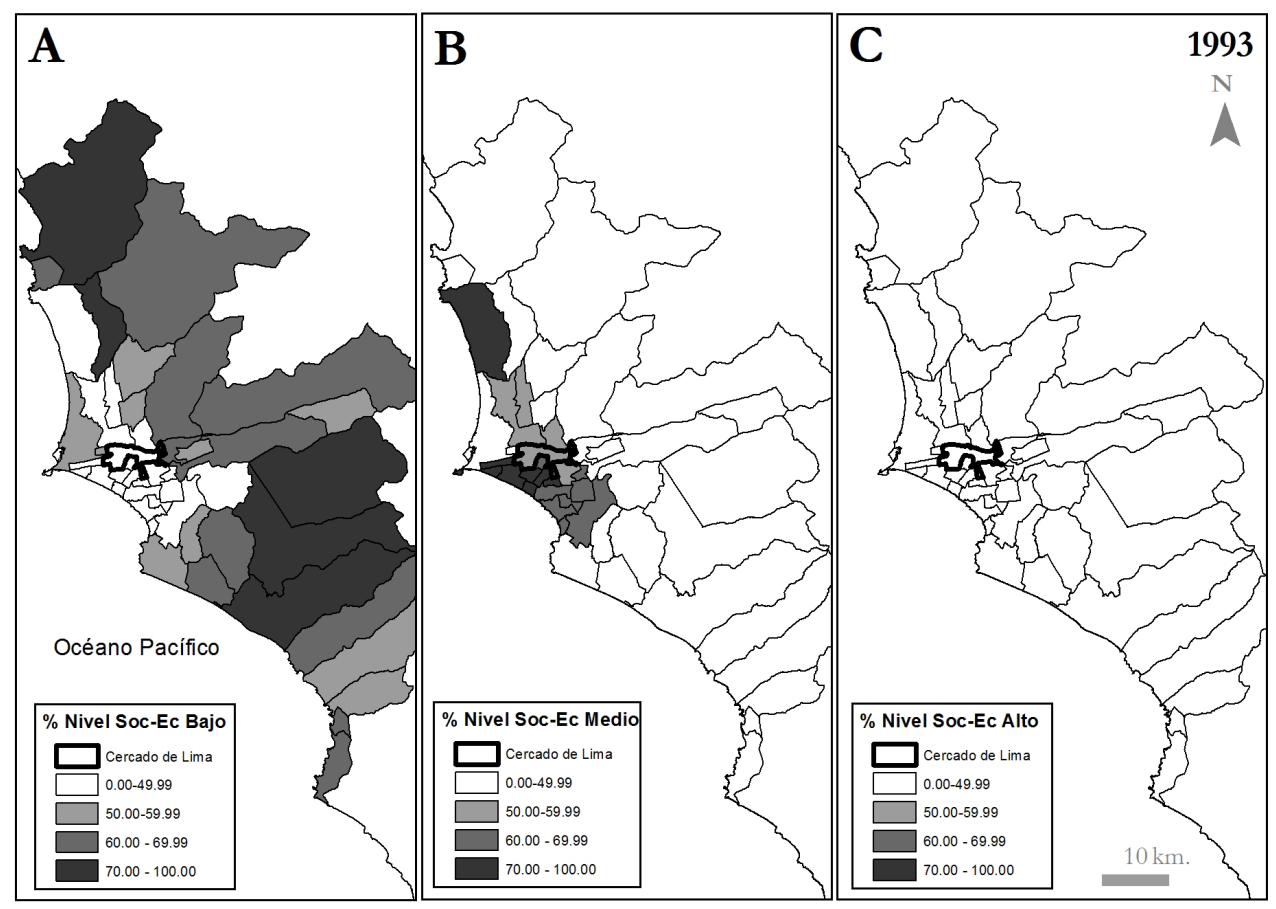

Fuente: Elaboración propia sobre la base del Censo Nacional 1993 (Base de Datos del Censo Nacional de Población y Vivienda 1993).

Hay que resaltar que, si bien el modelo es «segmentado», ello ocurre, en términos de mercado, entre aquellos que tienen recursos para participar en él y aquellos que no. No se ha detectado en la literatura que la discriminación racial o étnica sea per se un elemento generador de diferenciación espacial.

\section{DinÁMica de LA CIUDAd NEOLIBERAL}

La implementación de reformas neoliberales a fines de la década de 1980 en América Latina (inicios de la década de 1990 en el Perú) tuvo un impacto contundente en las ciudades de la región (Portes, Roberts y Grimson, 2006; Thiebert y Osorio, 2014). Se agrega a ello una disminución del crecimiento de las ciudades por migración para dar paso a un crecimiento vegetativo mucho menos acelerado que el de las décadas anteriores.

Centrándonos en Lima, varias consecuencias de las reformas neoliberales son relevantes para entender el cambio en el sistema de intereses y competencia por el lugar en la ciudad. Primero, una marcada estabilidad y crecimiento 
económicos que — unidos a la apertura de los mercados - dieron pie al crecimiento de las inversiones nacionales e internacionales en la ciudad. Dicho influjo de inversiones permitió la expansión de los sectores comercio y servicios, y más tarde la construcción inmobiliaria (Gonzales de Olarte, Del Solar y Del Pozo, 2011; Calderón, 2009; Benzús, 2018). Segundo, al igual que en el resto de la región, un marcado crecimiento de las clases medias y de su capacidad de consumo (Franco, Hopenhayn y León, 2010). En Lima, dicho crecimiento se asocia con la consolidación de un proceso de movilidad social de parte de los hijos y nietos de los antiguos migrantes. El resultado es una nueva clase media más diversa, no solo en términos de lugar de origen y color (Portocarrero, 1993; Pereyra, 2015), sino también en estilos de vida y patrones de consumo (Arellano y Burgos, 2010). En términos espaciales, en la medida en que varias familias experimentaron movilidad social ascendente, sus barrios de origen se tornaron en barrios de clase media (Arellano y Burgos, 2010). Tercero, un ingreso significativo de terrenos e inmuebles al mercado formal. Ello incluye no solo la formalización de terrenos y viviendas en las áreas periféricas o conos, sino también un nuevo periodo de construcción y densificación en la zona central de la ciudad para cubrir la nueva demanda de vivienda para la clase media (Calderón, 2009; Gonzales de Olarte et al., 2011). Cuarto, el paso de una estructura centralizada de la ciudad a una nueva estructura policéntrica (Gonzales de Olarte et al., 2011). Sin embargo, si bien surgen nuevos centros en las antiguas periferias de la ciudad, varios de los centros (antiguos y nuevos) se siguen concentrando en el área central de la ciudad. Es decir, se trata de un «policentrismo centralizado» (Gonzales de Olarte y Del Pozo, 2012, p. 49).

En esta nueva estructura, más acentuadamente «de mercado», cambian no solo los actores urbanos, sino también sus intereses. Como se mencionó, la ciudad sigue creciendo por migración, pero dicho crecimiento ya no es tan relevante como antes. Sin embargo, ello no significa que no exista pobreza. Por el contrario, la pobreza sigue siendo masiva. Lo nuevo en el escenario es que buena parte del mercado de suelos ya se encuentra formalizado. En este contexto, los más pobres tienen dos medios principales para adquirir vivienda sin comprarla: 1) densificar o tugurizar las viviendas de sus familiares; 2) invadir áreas no tomadas por el mercado o zonas periféricas aún más distantes (Barreda y Ramírez, 2004). Es decir, el modelo de "competencia segmentada» sigue existiendo. Sin embargo, la ciudad de hoy crece más por mecanismos de mercado formal — por la construcción y compra de nuevas viviendas — o informal — por el alquiler de cuartos o pisos en antiguas barriadas hoy consolidadas-. 
Respecto a los nuevos intereses residenciales, se mezclarían hoy en día criterios pragmáticos y de estatus. Son criterios estrictamente pragmáticos el buscar un lugar de residencia cercano (en distancia y tiempo) al trabajo, a servicios importantes (centros de estudios, comercio, entretenimiento) y a redes significativas (amigos y familia). El «policentrismo centralizado» de la ciudad incentiva que — sobre todo para las clases medias y altas — el área del «cono de alta renta» sea la más deseable, llevando a su densificación (Gonzales de Olarte y Del Pozo, 2012). Surgen también externalidades negativas como que, con la mayor densidad habitacional y con el crecimiento del parque automotor, el tiempo de desplazamiento en la ciudad acaba siendo aún mayor. Con ello, vivir en zonas alejadas de la ciudad supone un aumento significativo (en comparación con el periodo anterior) del tiempo de desplazamiento. De este modo, surgen incentivos fuertes para vivir en esta área de la ciudad; pero, por otro lado, mientras mayor es la presión sobre esta área, mayor el precio del metro cuadrado.

Pero se combinarían criterios de estatus con los estrictamente pragmáticos. En ese sentido, es importante evaluar hasta qué punto todas las áreas céntricas de la ciudad serían deseables o si existen estigmas asociados a algunas de ellas limitando su deseabilidad. En la medida en que las áreas más antiguas de invasión (aquellas cerca al centro de la ciudad) son hoy en día lugares "céntricos», estas ofrecerían un nuevo atractivo para las clases altas y medias tradicionales. Sería un argumento a favor de la tesis de la microsegregación la existencia de condominios cerrados exclusivos o casos de gentrificación en estas áreas a pesar de tener el estigma de ser antiguas barriadas o de acuerdo al origen de la población residente en estos lugares. Es posible que el estatus de estas áreas sea precisamente una limitante para las clases medias tradicionales y altas respecto a considerar estos lugares como posibles lugares de residencia. Al respecto, la literatura existente sugiere, más bien, que las ventajas de estas áreas estarían siendo aprovechadas no por las familias jóvenes de clase media tradicional, sino por los hijos y nietos de los vecinos originales de estos barrios (la "clase media emergente») que optan por construir segundos y terceros pisos en las viviendas de sus padres o por comprar viviendas o nuevos departamentos en estas áreas (Calderón, 2009).

Pero no solo cambia la estructura de intereses. Cambian también los recursos con los que cuentan los actores en este nuevo escenario. Como se mencionó, existe un crecimiento de las clases medias, las cuales se convierten en actores importantes en la dinámica residencial. A partir del aumento de la capacidad de gasto y de endeudamiento de este sector, sus intereses residenciales pueden materializarse en el mercado inmobiliario. En este contexto, ante el déficit de viviendas y la mayor capacidad de endeudamiento, surgen 
iniciativas privadas (como el mayor acceso a créditos hipotecarios) y públicoprivadas (principalmente el Fondo MiVivienda, creado en 1998), precisamente focalizadas hacia los sectores medios ${ }^{7}$. Para facilitar el crecimiento de estas empresas se redujeron los impuestos al sector construcción y se relajó la normatividad para otorgar licencias para la construcción de edificios (Calderón, 2009; Benzús, 2018). De este modo, surge un "boom inmobiliario» que contribuye significativamente a la densificación de la ciudad por medio de la construcción de edificios de departamentos (Calderón, 2009). Sin embargo, tenemos noticia que estos proyectos de vivienda se encuentran altamente concentrados en esta área de la ciudad (precisamente donde la clase media buscaba residir). Por ello, los investigadores en el tema sugieren que dicho boom debe haber contribuido a aumentar la segregación residencial (Calderón, 2009; Benzús, 2018).

En este contexto, gracias a las facilidades de crédito que se dan a un sector socioeconómico en particular (los sectores medios) y para la construcción de departamentos en un área en particular (los distritos de la zona central de la ciudad), proponemos describir esta nueva dinámica como de «competencia asistida». Como en el contexto anterior, sigue siendo una dinámica de "competencia» en la medida en que se trata de decisiones y estrategias residenciales individuales o familiares. Pero es una competencia «asistida» en la medida que existe esta intervención estatal para dinamizar el mercado inmobiliario con miras a favorecer directamente a los sectores medios. Como se mencionó, los lugares en donde se ubican los nuevos edificios de departamentos para estas familias se encuentran concentrados precisamente en los distritos del casco central de la ciudad, principalmente en los distritos tradicionales del "cono de alta renta» (tanto en algunos de los distritos más "populares» en esta zona como Cercado de Lima, Surquillo o el Callao, pero también en distritos muy tradicionales de clase media como San Miguel, Magdalena, Pueblo Libre, Lince, Jesús María, y algunos más exclusivos como Miraflores y Santiago de Surco), así como en los antiguos distritos donde se ubicaron varias invasiones, hoy mayormente consolidados (como San Martín de Porres y Comas en el Cono Norte, San Juan de Lurigancho y Ate en el Cono Este, y San Juan de Miraflores en el Cono Sur).

Hasta acá una rápida revisión de los nuevos procesos urbanos y los intereses que estos estarían forjando. Sigue el evaluar el impacto y la dirección que estos procesos estarían generando. Al respecto, en la investigación sobre ciudades en América Latina, se ha resaltado que el neoliberalismo, el aumento

De acuerdo a cálculos del programa, se encuentra un déficit de 100000 viviendas para la clase media (ver Calderón, 2009). 
de la criminalidad y las nuevas tecnologías de transporte (avenidas rápidas y by-passes) y de seguridad (cámaras de vigilancia, empresas de seguridad privada), han incentivado la aparición de nuevas formas de localización residencial en las que los sectores altos tienden a acercarse espacialmente a los sectores populares, sin que ello signifique necesariamente que estos grupos se integren socialmente (Caldeira, 2000; Janoschka, 2002; Thiebert y Osorio, 2014). De este modo, ha llamado la atención la aparición masiva de fenómenos como los condominios cerrados para las élites ubicados en zonas pobres de la ciudad (Caldeira, 2000; Cáceres y Sabatini, 2004), así como casos de gentrificación (Lees, Chin y López Morales, 2015). Es así que, en este nuevo contexto, se sugiere que se superpone al modelo previo de "segregación de gran escala» un nuevo modelo de «segregación de pequeña escala», en donde ricos y pobres viven cerca unos de los otros, pero manteniendo o erigiendo barreras físicas y sociales (Caldeira, 2000). Ahora pasaremos a discutir el alcance de dicho nuevo modelo para el caso de Lima.

\section{LA CONSOLIDACIÓN DE LA SEGREGACIÓN RESIDENCIAL DE GRAN ESCALA}

En esta sección nos interesa evaluar el resultado del conjunto de incentivos inducidos para los actores a partir de grandes procesos urbanos y, con ello, evaluar la relevancia de la tesis de la microsegregación para el caso de Lima. La evaluación general es que, si bien hay información divergente respecto a la intensidad de la segregación residencial para los sectores más pobres, el modelo de segregación residencial de gran escala se ha reforzado en las últimas décadas. Como muestra el Cuadro 1, la segregación entre los censos 1993 y 2007 se ha incrementado en distinto grado (con variaciones por tipo de índice) para todos los NSE. Debe notarse también que los grupos que experimentan aumentos más significativos en su segregación son los NSE bajo y (sobre todo) alto. Asimismo, los Mapas 2A, 2B y 2C muestran una representación gráfica de este fenómeno (compárese con los Mapas 1A, 1B y 1C, respectivamente).

En el caso del grupo de NSE bajo (Mapa 2A), la segregación sigue la dinámica de la etapa anterior: altas concentraciones de pobreza en los distritos periféricos de la ciudad formando grandes bloques principalmente en los Conos Norte y Sur. Es necesario señalar dos puntos respecto al patrón de ubicación de este grupo. 1) Respecto a la composición más fina de los llamados conos, Fernández de Córdova et al. (2016) señalan (sobre la base de información del Censo 2007 a nivel zona), que sus áreas más antiguas son visiblemente heterogéneas dado el proceso de consolidación de estas áreas, así como por el proceso de movilidad social ascendente de algunos de estos residentes. 
Cuadro 1. Índices de segregación residencial por niveles socioeconómicos, 1993 y 2007

\begin{tabular}{lll}
\hline & 1993 & 2007 \\
\hline Disimilaridad $(D)$ & & \\
$\quad$ Bajo & 0.31 & 0.44 \\
Medio & 0.23 & 0.37 \\
$\quad$ Alto & 0.64 & 0.89 \\
Aislamiento $\left(P^{*}\right)$ & & \\
$\quad$ Bajo & 0.56 & 0.59 \\
Medio & 0.52 & 0.61 \\
Alto & 0.18 & 0.49 \\
Aglomeración $(I$ de Moran $)$ & & \\
Bajo & 0.15 & 0.21 \\
Medio & 0.12 & 0.12 \\
Alto & 0.14 & 0.14 \\
\hline
\end{tabular}

Fuente: Elaboración propia sobre la base de los datos de los censos 1993 (Base de Datos del Censo Nacional de Población y Vivienda 1993) y 2007 (INEI, 2009).

\section{Mapa 2. Población por nivel socioeconómico, Lima 2007}

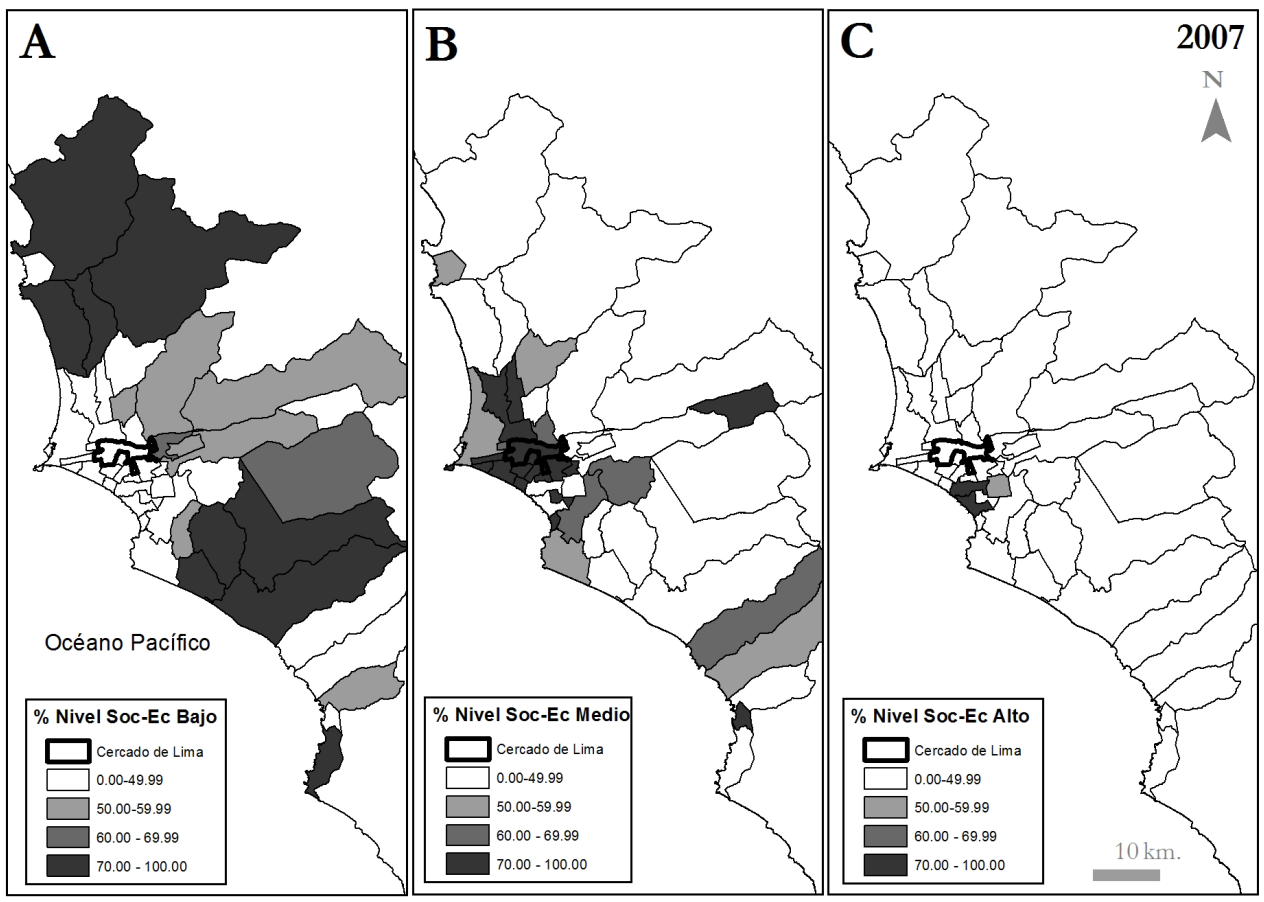

Fuente: Elaboración propia sobre la base del Censo Nacional 2007 (INEI, 2009). 
Aunque, como señalamos más adelante, si bien dicha heterogeneidad existe, agregamos que existe una fuerte tendencia a que los distritos de Lima se mantengan estables en términos de la composición de la población por NSE, o a reducir su nivel de heterogeneidad para ser hoy en día más homogéneos (ver Anexo 1). 2) Respecto al grupo más pobre, Herrera et al. (2016) señalan que su segregación ha disminuido entre los dos últimos censos (ello sobre la base de

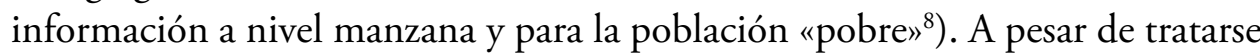
de una diferencia sobre la base de la definición de grupos («bajo» en un caso, «pobre» en otro), nuestros datos concuerdan con los suyos al señalar que existe una tendencia a que la población de NSE más bajo se concentra masivamente y sigue ubicándose en las áreas más distantes formando grandes aglomeraciones periféricas.

Sin embargo, debe llamar la atención que la dinámica más significativa ocurre en el área central de la ciudad, animada tanto por los NSE medios y altos (grupos poco atendidos por la investigación en segregación residencial). Lo que puede observarse es la consolidación de esta área como una nueva zona de concentración de población de NSE medio (Mapa 2B). Dos grandes dinámicas se pueden resaltar. Primero, la antigua «zona de alta renta» (sur-este de la ciudad) ha atraído a más población de NSE medio, haciendo de esta una zona segregada consolidada de este grupo. Segundo, el resto del casco central (incluyendo los distritos más antiguos del Cono Norte), ha pasado de ser un área relativamente heterogénea a ser también una gran concentración de población de NSE medio. Esta evidencia es consistente con la idea que esta área, debido al modelo del «descentralismo centralizado», es hoy en día bastante deseada, pero no solo en el área sur-este, como en el periodo anterior, sino ahora en su totalidad del área central, incluyendo a los distritos más antiguos del Cono Norte.

Finalmente, en el caso del grupo de NSE alto (Mapa 2C), se encuentra que este grupo tiende a aislarse con mayor fuerza y de forma más concentrada que antes. Debe resaltarse que si bien la tendencia anterior era a que este grupo se desplazara hacia zonas cada vez más lejanas - formando islotes en distritos como San Borja o La Molina (ver Pereyra, 2006)—, ahora este grupo también se concentra de forma muy notoria en Miraflores y San Isidro (dos distritos de la zona central) y en San Borja (más hacia el sur-este), formando por primera vez una gran zona exclusiva de la ciudad. Dicha concentración tiene sentido en el contexto del modelo del "policentrismo centralizado", en el cual la distancia espacial de los centros urbanos tiene costos altos en términos de tiempo de

8 Entiéndase por «pobre» al 25\% de menores ingresos estimados (Herrera et al., 2016, p. 46). 
desplazamiento, por lo que este grupo tiende a acercarse nuevamente al área central de la ciudad. Sin embargo, de forma contraria a la tesis clásica de la microsegregación, el grupo del NSE alto tiende a formar zonas exclusivas, no en distritos pobres, sino en estos distritos que ya eran medianamente exclusivos y/o que contaban con concentraciones previas de población de NSE medio. Es decir, si el NSE alto construye condominios cerrados o se recluye en edificios exclusivos, ello ocurre principalmente en distritos que previamente tenían una alta presencia de NSE alto y medio; no en distritos de población de NSE bajo, como sugiere el modelo clásico de microsegregación.

\section{Conclusiones}

La evidencia estudiada muestra que el modelo de segregación residencial de gran escala no se ha debilitado, sino que más bien se ha fortalecido. Lima, hoy en día, se caracteriza por mostrar grandes concentraciones relativamente homogéneas de población por NSE. Si bien esto no niega que existan casos de condominios cerrados exclusivos en distritos pobres o casos de gentrificación, su presencia no es significativa como para dar un giro a la tendencia principal que es la de la consolidación de la segregación de gran escala. De hecho, no solo los distritos concentran cada vez más población de un mismo NSE, sino que además hay menos distritos heterogéneos en 2007 que en 1993 (ver Anexo 1).

En este artículo se sugiere que esta dinámica de la segregación residencial es consecuente con un nuevo modelo de elección y competencia residencial que obedece principalmente a motivos pragmáticos. La proximidad a los centros urbanos hace que algunos lugares, principalmente aquellos en el casco central de la ciudad, sean los más deseados, incluso por el grupo del NSE alto que antes buscaba más bien alejarse de la ciudad. Sin embargo, esta dinámica de consolidación de la segregación de gran escala parece sugerir que esta también fue orientada por un criterio de homofilia (en este caso en términos de NSE). Es decir, la nueva dinámica urbana no juntó extremos de población, sino grupos cercanos en términos de NSE: la población de NSE alto se ubicó en distritos de NSE medio, y la población de NSE medio se ubicó en distritos de NSE medio o al menos heterogéneos. Asimismo, el boom inmobiliario reforzó está dinámica facilitando la compra de departamentos para el NSE medio en zonas deseables para este grupo. La consecuencia fue la consolidación de un gran bloque segregado de población de NSE medio en la zona central de la ciudad. Sin embargo, como sugiere la literatura, es posible que el criterio de estatus siga siendo importante en esta decisión: la clase media tradicional 
seguiría prefiriendo los distritos tradicionales de clase media (la zona sur-este); pero la llamada «nueva clase media» podría estar ubicándose tanto en la zona norte de esta área central como en el sur-este.

Finalmente, algunos puntos respecto al "conflicto» en la ciudad. Ante todo, debe resaltarse que, con el debilitamiento del proceso de crecimiento por migración e invasión de terrenos, el conflicto en torno a la ciudadanía social —aquel por servicios básicos, propiedad, salud, educación, etcétera (ver Roberts, 1978) — deja de ser el más visible. Ello no significa que este tipo de conflicto deje de ser importante, sino que pasa a ser uno entre otros. Cobran centralidad en esta nueva etapa otros conflictos, esta vez en otras zonas de la ciudad o en la ciudad completa. Así, la densificación del área central de la ciudad ha ocurrido a expensas de relajar criterios de planificación (Calderón, 2009; Benzús, 2018). Surgen entonces nuevos conflictos locales o distritales en esta zona a partir de las presiones para reducir o privatizar los espacios públicos o por ampliar calles y avenidas para la circulación de automóviles. Se agrega una dinámica nueva que es que algunas de estas áreas empiezan a tener valor comercial, generando la aparición de comercios pequeños y medianos, malls y edificios de oficinas. Surgen así nuevos fenómenos en estas áreas, como el aumento del potencial comercial de los vecindarios, el cambio de zonificación y hasta la pérdida de población de algunos distritos (precisamente algunos de los distritos que concentran grandes nodos urbanos como Miraflores y San Isidro). Finalmente, surgen nuevos conflictos en torno a la calidad de vida y los servicios colectivos metropolitanos. Empiezan a cobrar relevancia movimientos a favor de la planificación, del cuidado del medio ambiente, del espacio público, por un transporte eficiente, contra la criminalidad, y también reivindicativos de estilos de vida y por la tolerancia. Debe llamar la atención que ya no son movimientos barriales o que involucren a un solo tipo de actor, sino que son movimientos metropolitanos y que integran a distintos tipos de actores.

\section{REFERENCIAS}

Abramo, P. (2012). La ciudad com-fusa: Mercado y producción de la estructura urbana en las grandes metrópolis latinoamericanas. EURE, 38(114), 35-69. https://doi.org/10.4067/ S0250-71612012000200002

Arellano, R. y Burgos, D. (2010). Ciudad de los Reyes, de los Chávez, de los Quispe. Lima: Planeta.

Barreda, J. y Ramírez, D. (2004). Lima: Consolidación y expansión de una ciudad popular. En Desco (ed.), Las ciudades en el Perú hoy (pp. 199-218). Lima: Desco.

Benavides, M. y León, J. (2015). «Segregación urbana y violencia: Un análisis en contextos de pobreza de cinco distritos de Lima metropolitana». Ponencia presentada en el Seminario 
Internacional Las ciudades de América Latina: Entre el mediano y largo plazo: Planificación, centralidad y desigualdad. Lima, 10 y 11 de diciembre.

Benavides, M., León, J., Paredes, A. y La Riva, D. (2019). Capital social y logro ocupacional en contextos de segregación. Documento de investigación No. 95. Lima: Grade. Recuperado de http:// www.grade.org.pe/wp-content/uploads/GRADEdi95.pdf

Benzús, V. (2018). Densificación (no) planificada de una metrópoli: El caso del área metropolitana de Lima 2000-2014. INVI, 33(92). https://doi.org/10.4067/S0718-83582018000100009

Cáceres, G. y Sabatini, F. (eds.) (2004). Barrios cerrados en Santiago de Chile: Entre la exclusión y la segregación residencial. Santiago: Lincoln Institute of Land Policy - Pontificia Universidad Católica de Chile.

Caldeira, T. (2000). City of Walls: Crime, Segregation and Citizenship in Sao Paulo. Berkeley - Los Angeles: University of California Press.

Calderón, J. (2005). La ciudad ilegal: Lima en el siglo XX. Lima: Universidad Nacional Mayor de San Marcos.

Calderón, J. (2009). El efecto Mivivienda: Política de vivienda para la clase media y diferenciación social. Ecuador Debate, (76).

Castells, M. (1974). La cuestión urbana. Madrid: Siglo XXI.

Charles, C. (2006). Won't You Be My Neighbor: Race, Class and Residence in Los Angeles. Nueva York: Russell Sage Foundation.

Driant, J. C. (1991). Las barriadas de Lima: historia e interpretación. Lima: Instituto Francés de Estudios Andinos (IFEA)-Desco. https://doi.org/10.4000/books.ifea.6962

Fernández de Córdova, G. (2012). Nuevos patrones de segregación socio-espacial en Lima y Callao 1993-2007: Nuevos desafíos para la metrópoli. Cuaderno de trabajo No. 15. Lima: Facultad de Arquitectura y Urbanismo de la Pontificia Universidad Católica del Perú.

Fernández de Córdova, G., Fernández Maldonado, A. M. y Del Pozo, J. M. (2016). Recent Changes in the Patterns of Socio-Spatial Segregation in Metropolitan Lima. Habitat International, 54. https://doi.org/10.1016/j.habitatint.2015.08.016

Franco, R., Hopenhayn, M. y León, A. (eds.) (2010). Las clases medias en América Latina: Retrospectiva y nuevas tendencias. México D.F.: Cepal - Siglo XXI.

Galster, G. y Killen, S. (1995). The Geography of Opportunity: A Reconnaissance and Conceptual Framework. Housing Policy Debate, 6(1). https://doi.org/10.1080/10511482.1995.9521180

Gilbert, A. (1998). The Latin American City. Londres: The Latin American Bureau.

Gonzales de Olarte, E., Del Solar, V. y Del Pozo, J. M. (2011). Lima metropolitana después de las reformas neoliberales: Transformaciones económicas y urbanas. En C. de Mattos y W. Ludeña (eds.), Lima-Santiago: Reestructuración y cambio metropolitano (pp. 135-176). Lima-Santiago: Pontificia Universidad Católica de Chile - Pontificia Universidad Católica del Perú.

Gonzales de Olarte, E. y Del Pozo, J.M. (2012). ¿Lima, una ciudad policéntrica?: Un análisis a partir de la localización del empleo. Investigaciones Regionales, (23), 29-52.

Harvey, D. 1978. The Urban Process under Capitalism: A Framework of Analysis. International Journal of Urban and Regional Research, 2(1-3). https://doi.org/10.1111/j.1468-2427.1978. tb00738.x

Herrera, J., Benavides, M., Etesse, M. y Letoutier, M. (2016). Trends in Segregation and Poverty in Metropolitan Lima. NOPOOR Project (Documento sin publicar).

Instituto Nacional de Estadística e Informática - INEI (2009). Planos estratificados de Lima metropolitana a nivel manzana. Lima: INEI.

Janoschka, M. (2002). El nuevo modelo de ciudad: fragmentación y privatización. EURE, 28(85). https://doi.org/10.4067/S0250-71612002008500002 
Katzman, R. (2001). Seducidos y abandonados: el aislamiento social de los pobres urbanos. Revista Cepal, 75, 171-189. https://doi.org/10.18356/16a0b21c-es

Lees, L., Chin, H.B. y López-Morales, E. (2015). Global Gentrifications: Uneven Development and Displacement. Bristol: Polity Press. https://doi.org/10.1332/policypress/ 9781447313472.001 .0001

Logan, J. y Molotch, H. (1987). Urban Fortunes: The Political Economy of Place. Berkeley - Los Angeles: University of California Press.

Marques, E. (2016). Urban Poverty, Segregation and Social Networks in Sao Paulo and Salvador, Brazil. International Journal of Urban and Regional Research, 39(6), 1067-1083. https://doi. org/10.1111/1468-2427.12300

Massey, D. y Denton, N. (1993). American Apartheid: Segregation and the Making of the Underclass. Cambridge MA: Harvard University Press.

Parker, D. (1995). Los pobres de la clase media: Estilo de vida, consumo e identidad en una ciudad tradicional. En A. Panfichi y F. Portocarrero (comps.), Mundos interiores: Lima 1850-1950 (pp. 161-185). Lima: Universidad del Pacífico.

Pereyra, O. (2006). Forma urbana y segregación residencial en Lima. Debates en Sociología, 31, 69-106. http://revistas.pucp.edu.pe/index.php/debatesensociologia/article/view/2704

Pereyra, O. (2015). Contemporary Latin American Middle Class: The Case of San Felipe. Lanham: Lexington Books.

Peters P. y Skop, E. (2007). Socio-Spatial Segregation in Metropolitan Lima, Peru. Journal of Latin American Geography, 6(1), 141-171. https://doi.org/10.1353/lag.2007.0009

Peters, P. y Hall, G. B. (2004). Evaluation of Education Quality and Neighborhood Wellbeing: A Case Study of Independencia. International Journal of Educational Development, 24(1), 85-102. https://doi.org/10.1016/j.ijedudev.2003.10.003

Portes, A., Roberts, B. y Grimson, A. (2006). Ciudades latinoamericanas: Un análisis comparativo en el umbral del nuevo siglo. Buenos Aires: Prometeo.

Portocarrero, G. (1993). Introducción: Ajuste de cuentas: las clases medias en el trabajo de Tempo. En G. Portocarrero (ed.), Los nuevos limeños: sueños, fervores y caminos en el mundo popular (pp. 9-37). Lima: Sur.

Riofrío, G. (1991). Producir la ciudad (popular) de los 90. Entre el Estado y el mercado. Lima: Desco.

Roberts, B. (1978). Cities of Peasants: The Political Economy of Urbanization in the Third World. Beverly Hills - Londres: Sage Publications.

Roberts, B. y Wilson, R. (eds.) (2009). Segregation and Urban Governance in the Americas. Nueva York: Palgrave Mcmillan. https://doi.org/10.1057/9780230620841

Sabatini, F. (2003). La segregación social del espacio en las ciudades de América Latina. Serie Azul, No. 35. Santiago de Chile: Pontificia Universidad Católica de Chile.

Sabatini, F., Wormald, G. y Rasse, A. (eds.) (2013). Segregación de la vivienda social: Ocho conjuntos en Santiago, Concepción y Talca. Santiago: Pontificia Universidad Católica de Chile.

Sampson, R., Morenoff, J. y Ganon-Rowley, T. (2002). Assessing 'Neighborhood Effects': Social Processes and New Directions in Research. Anual Review of Sociology, 28, 443-478. https://doi. org/10.1146/annurev.soc.28.110601.141114

Schelling, T. (1971). Dynamic Models of Segregation. Journal of Mathematical Sociology, 1, 143186. https://doi.org/10.1080/0022250X.1971.9989794

Telles, E. (1992). Residential Segregation by Skin Color in Brazil. American Sociological Review, 57(2), 186-197. https://doi.org/10.2307/2096204

Thiebert, J. y Osorio, G. (2014). Urban Segregation and Metropolitics in Latin America: The Case of Bogotá, Colombia. International Journal of Urban and Regional Research, 38(4), 1319-1343. https://doi.org/10.1111/1468-2427.12021 
Tilly, C., Moss, P., Kirschenman, J. y Kennelly, I. (2001). Space as Signal: How Employers Perceive Neighborhoods in Four Metropolitan Labor Markets. En A. O'Connor, C. Tilly y L. D. Bobo (eds.), Urban Inequality: Evidence from Four Cities (pp. 304-338). Nueva York: Russell Sage Foundation.

Wacquant, L. (2008). Urban Outcasts: A Comparative Sociology of Advanced Marginality. Malden: Polity Press.

Wilson, J.W. (1987). The Truly Disadvantaged: The Inner City, the Underclass and Public Policy. Chicago: The University of Chicago Press.

Wilson, J.W. (1997). When Work Disappears: The World of the New Urban Poor. Nueva York: Vintage. 


\section{Anexo 1}

Matriz de movilidad de distritos por NSE, 1993-2007

\begin{tabular}{|c|c|c|c|c|}
\hline & Bajo* 1993 & Medio* $^{*} 1993$ & Alto* 1993 & Mixto** $^{*} 1993$ \\
\hline $\begin{array}{l}\text { Bajo* } \\
2007\end{array}$ & $\begin{array}{l}\text { Ancón } \\
\text { Carabayllo } \\
\text { Cieneguilla } \\
\text { El Agustino } \\
\text { Lurín } \\
\text { Pachacamac } \\
\text { Pucusana } \\
\text { Puente Piedra } \\
\text { Villa El Salvador } \\
\text { Villa María del } \\
\text { Triunfo }\end{array}$ & Ventanilla & -- & Ate \\
\hline $\begin{array}{l}\text { Medio* } \\
2007\end{array}$ & $\begin{array}{l}\text { Punta Hermosa } \\
\text { Santa María del Mar }\end{array}$ & $\begin{array}{l}\text { Barranco } \\
\text { Breńa } \\
\text { Jesús María } \\
\text { Lince } \\
\text { Magdalena } \\
\text { Pueblo Libre } \\
\text { San Luis } \\
\text { San Miguel } \\
\text { Santiago de Surco } \\
\text { Surquillo } \\
\text { Bellavista } \\
\text { La Perla } \\
\text { La Punta }\end{array}$ & -- & $\begin{array}{l}\text { Cercado } \\
\text { Chaclacayo } \\
\text { La Molina } \\
\text { La Victoria } \\
\text { Los Olivos } \\
\text { Rímac } \\
\text { San Martín de Porres } \\
\text { Carmen de la Legua }\end{array}$ \\
\hline $\begin{array}{l}\text { Alto* } \\
2007\end{array}$ & --- & $\begin{array}{l}\text { Miraflores } \\
\text { San Isidro }\end{array}$ & -- & -- \\
\hline $\begin{array}{l}\text { Mixto** } \\
2007\end{array}$ & $\begin{array}{l}\text { San Juan de } \\
\text { Lurigancho } \\
\text { Santa Rosa }\end{array}$ & San Borja & --- & $\begin{array}{l}\text { Chorrillos } \\
\text { Comas } \\
\text { Independencia } \\
\text { Lurigancho } \\
\text { Punta Negra } \\
\text { Santa Anita } \\
\text { San Bartolo } \\
\text { San Juan de Miraflores } \\
\text { Callao }\end{array}$ \\
\hline
\end{tabular}

* Se entiende que un distrito es de un NSE si la mayoría de su población (60\% o más) pertenece a dicho NSE.

** Se entiende que un distrito es mixto si ningún grupo de NSE alcanza más del 59,99\% de su población.

Fuente: Elaboración propia sobre la base de los datos de los Censos Nacionales 1993 y 2007. 